\title{
Adaptive consensus for high-order unknown nonlinear multi-agent systems with unknown control directions and switching topologies
}

\author{
Mohammad Hadi Rezaei ${ }^{\text {a,b }}$, Meisam Kabiri ${ }^{\text {a,b }}$, Mohammad Bagher Menhaj a,b,* \\ a Department of Electrical Engineering, Amirkabir University of Technology (Tehran Polytechnic), Tehran, Iran \\ ${ }^{\mathrm{b}}$ The Center of Excellence on Control and Robotics, Department of Electrical Engineering, Amirkabir University of Technology (Tehran \\ Polytechnic), Tehran, Iran
}

\section{A R T I C L E I N F O}

\section{Article history:}

Received 7 January 2018

Revised 4 April 2018

Accepted 29 April 2018

Available online $\mathrm{xxx}$

\section{Keywords:}

Consensus

High-order nonlinear multi-agent systems

Unknown control directions

Input saturation

Switching topologies

Unknown dynamics

\begin{abstract}
A B S T R A C T
In this paper, we provide a comprehensive assessment of the consensus of high-order nonlinear multi-agent systems with input saturation and time-varying disturbance under switching topologies. The control directions and model parameters of agents are supposed to be unknown. Our approach is based on transforming the problem of consensus for a network that consists of high-order nonlinear agents to that of perturbed first-order multi-agent systems. The unknown part of dynamics is cancelled using radial basis neural networks. Nussbaum gains and auxiliary systems are respectively employed to overcome the unknown input direction and the saturation. Adaptive sliding mode control is used to compensate for the time-varying disturbance and the imperfect approximation of the developed neural network as well. Through Lyapunov analysis, it is shown that the overall closed-loop system maintains asymptotic stability. Finally, our approach is applied to a group of multiple single-link flexible joint manipulators to highlight better its merit.
\end{abstract}

(c) 2018 Published by Elsevier Inc.

\section{Introduction}

Advances in networked cyber-physical systems and embedded systems technology have created an increasing interest among the control community to study multi-agent systems (MASs). The control techniques developed so far for MASs enable us to apply resilient, cheap, and flexible methodologies to diverse cooperative tasks in many domains including maintenance, surveillance, reconnaissance, search and rescue mission, cooperative construction, and manipulation [6,18,28,31,47].

Consensus is a fundamental cooperative task in MASs where all the agents in a team are supposed to agree on a certain value of interest while each agent updates its states merely on the basis of its own states and the local information from its neighbors. Consensus has applications in a variety of domains, including cooperation of network sensors [35], decision making [26], motion coordination of unmanned aerial vehicles (UAVs) $[23,40]$ and autonomous underwater vehicles (AUVs) [2], attitude synchronization in spacecraft [48], flocking control [33], and load sharing in microgrids [12].

Early studies of consensus mainly focused on the cooperation of agents with first- and second-order dynamics $[20,27,29,34]$. In [34], the problem of average consensus for first-order integrators under switching topologies and identical time delays was fully developed. The authors in [20] fully discussed the consensus problem of second-order system

\footnotetext{
* Corresponding author.

E-mail addresses: hadi.rezaei@aut.ac.ir (M.H. Rezaei), mk13@aut.ac.ir (M. Kabiri), menhaj@aut.ac.ir (M.B. Menhaj).
} 
models under jointly-connected switching topologies based on a space decomposition technique. In [29], necessary and sufficient conditions were derived to guarantee consensus in a network of second-order systems over directed topology with a uniform constant delay.

However, first- and second-order kinematics fail to model many practical systems described by high-order differential equations. Hence, several studies concentrated on the consensus problem for linear high-order dynamics [4,11,39,41]. Techniques such as feedback linearization can be used to convert nonlinear systems to linear ones. The perfect cancellation of the nonlinearities requires having an exact model for the system which is not feasible in reality. Therefore, applying the results of linear MASs to unknown nonlinear MASs is not straightforward. However, despite its great importance, there are rather few studies dedicated to the consensus of high-order nonlinear systems $[19,21,37,38,45,46]$. For example, the authors in [37] developed a consensus framework for a network consisting of uncertain high-order nonlinear systems under jointly-connected switching topologies. The work of [21], investigates consensus problem for high-order stochastic nonlinear systems under fixed topology. In [19], the leader-following consensus for nonlinear homogenous MASs with network induced delays is studied.

Cooperative control of high-order nonlinear MASs can be even more challenging in the presence of input saturation. This practical concern results from the physical constraints of actuators. This issue has been studied in [7,36,43,44]. The study of [36] was dedicated to the semi-global bipartite consensus of general linear MASs with switching topologies. In [43], leader-following output consensus of linear discrete-time MASs subject to actuator saturation and external disturbances was examined. A leader-follower framework for consensus of a group of linear MASs with input saturation was established in [44].

All the aforementioned studies shared the assumption that the control directions are known. Nevertheless, in some practical situations, the controlling effect is not accessible. This issue is already addressed for a single system by using the Nussbaum-type function initially introduced in [32]. Tackling this issue for control of MASs is challenging due to the fact that each agent Nussbaum gain parameter may move in a different direction which impedes using the usual method of contradiction in the establishment of the stability of the overall system [13]. Recently some studies have appeared to overcome this challenge. The authors in [3] investigated adaptive consensus problem of first-order and second-order linearly parameterized systems where the control directions are assumed to have known lower and upper bounds. The problem of adaptive output regulation in the presence of unknown identical control direction was addressed in $[13,30]$ in which the need for prior knowledge of the lower and upper bounds was removed. Researchers in $[3,13,30]$ investigated the special case where all the control directions of the subsystems are identical. Although this condition was relaxed in [1], it still relies on knowing some of the control directions.

The above-mentioned facts motivated us to address the problem of consensus for high-order unknown nonlinear systems with input saturation, time-varying disturbance, and unknown control direction under switching interaction topologies. We develop an approach that converts the problem into the consensus of first-order MASs with bounded perturbation terms and then employs a properly developed stabilizing controller. Radial basis function neural networks are used to approximate the unknown nonlinear part of the system dynamics, while their update rules are derived based on the Lyapunov analysis. In order to deal with the unknown control direction, the Nussbaum-type function is utilized. An auxiliary system is also implemented for each agent to compensate for the input saturation while the effect of the time-varying disturbance and the approximation error of the neural network is counteracted by an adaptive sliding mode control scheme.

Compared to the most relevant study [37], our approach takes into account the effects of both unknown control direction and input saturation. Besides, in the work of [37], the control parameters were determined in such a way that a linear matrix inequality (LMI), which is dependent on the number of agents, should hold. Hence, growing the number of agents in the network would increase the computational burden. To the best of our knowledge, no studies have yet considered the problem at hand in the presence of the all aforementioned practical issues. For example, in [21,37,38,45], the unknown control direction was not addressed. Existing approaches that cope with this difficulty $[1,3,13,30]$, not only neglected the effects of actuator saturation and switching topologies but also required the input directions to satisfy some limiting conditions. As a case in point, in [3], it was supposed that the upper and lower bounds of control effects are known. In [3,13,30], it was assumed that all control directions have an identical sign and in [1] some part of the control coefficients was considered to be known. In Table 1, the differences of our method with those presented in other relevant studies are highlighted. The contributions of this paper can be summarized as follows:

- Consensus for a class of general complex high-order nonlinear MASs with unknown nonlinearity and disturbance is studied.

- The consensus is achieved asymptotically in the presence of jointly connected topology for undirected graphs and uniformly jointly quasi-strongly connected and balanced topology for directed graphs.

- The impact of the actuator saturation in MASs is effectively handled.

- The proposed approach is capable of completely tackling unknown control effects where it allows control effects to have non-identical signs and the need for the knowledge of the boundaries of the control coefficients is removed.

The rest of the paper is organized as follows. In Section 2, required concepts from graph theory and notations are presented. The problem is formulated in Section 3. In Section 4, the control design and main results are presented. Simulations are given in Section 5 and finally, the paper is concluded in Section 6 .

Please cite this article as: M.H. Rezaei et al., Adaptive consensus for high-order unknown nonlinear multi-
agent systems with unknown control directions and switching topologies, Information Sciences (2018),
https://doi.org/10.1016/j.ins.2018.04.089


M.H. Rezaei et al./Information Sciences 000 (2018) 1-14

Table 1

Comparisons between our approach and those proposed in the other existing relevant studies in the literature

\begin{tabular}{|c|c|c|c|c|c|}
\hline References & $\begin{array}{c}\text { System } \\
\text { under } \\
\text { consideration }\end{array}$ & $\begin{array}{c}\text { Switching } \\
\text { topology }\end{array}$ & $\begin{array}{c}\text { Input } \\
\text { saturation }\end{array}$ & $\begin{array}{c}\text { Unknown } \\
\text { input direction }\end{array}$ & $\begin{array}{c}\text { Disturbance } \\
\text { or } \\
\text { uncertainties }\end{array}$ \\
\hline$[21,4]$ & Linear system & No & No & No & No \\
\hline$[38,39]$ & $\begin{array}{c}\text { High-order } \\
\text { nonlinear system }\end{array}$ & Yes & No & No & Yes \\
\hline$[45,7]$ & Linear system & No & Yes & No & Yes \\
\hline$[46]$ & $\begin{array}{c}\text { High-order } \\
\text { nonlinear system }\end{array}$ & No & No & No & Yes \\
\hline [44] & Linear system & No & Yes & No & Yes \\
\hline$[37]$ & Linear system & No & Yes & No & No \\
\hline$[3]$ & $\begin{array}{l}\text { first- and } \\
\text { second-order } \\
\text { systems }\end{array}$ & No & No & Yes & Yes \\
\hline$[13]$ & $\begin{array}{c}\text { High-order } \\
\text { nonlinear system }\end{array}$ & No & No & Yes & Yes \\
\hline$[31]$ & Nonlinear system & No & No & Yes & Yes \\
\hline$[1]$ & $\begin{array}{c}\text { High-order } \\
\text { nonlinear system }\end{array}$ & No & No & Yes & Yes \\
\hline $\begin{array}{l}\text { Proposed } \\
\text { approach }\end{array}$ & $\begin{array}{c}\text { High-order } \\
\text { nonlinear system }\end{array}$ & Yes & Yes & Yes & Yes \\
\hline
\end{tabular}

\section{Preliminaries}

In this section, we introduce the notations and the graph theory terminology which will be used throughout the paper.

\subsection{Notations}

Throughout this paper, symbols $\mathbb{R}$ and $\mathbb{R}^{+}$denote the set of reals and positive reals, respectively. sgn(.) expresses the sign function. $\mathbf{1}_{N}$ is a column vector of $N$ elements all equal to one. $\boldsymbol{0}_{N}$ denotes a column vector of $N$ with zero entries. $L_{\infty}$ presents the space of bounded signals. $x(t) \in L_{1}$ means that $\int_{0}^{\infty}|x(t)| \mathrm{d} t<\infty$.

\subsection{Graph theory}

The interaction among $N$ agents is represented by an undirected or directed graph $G(\mathcal{V}, \mathcal{E}, A)$ with a node set $\mathcal{V}=$ $\{1, \ldots, n\}$, edge set $\mathcal{E} \in\{\mathcal{V} \times \mathcal{V}\}$, and adjacency matrix $A=\left[a_{i j}\right] \in \mathbb{R}^{n \times n}$. The adjacency matrix is defined such that the diagonal entries are equal to zero $\left(a_{i i}=0\right)$ and the off-diagonal entries are $a_{i j}>0$ if $(i, j) \in \mathcal{E}$ and $a_{i j}=0$, otherwise. For undirected graphs, we also have $a_{i j}=a_{j i}$. The set of neighbors of node $v_{i}$ is $\mathcal{N}_{i}=\left\{v_{j} \in \mathcal{V} \mid\left(v_{j}, v_{i}\right) \in \mathcal{E}\right\}$. The Laplacian matrix $\mathcal{L}=\left[l_{i j}\right]$ is defined as

$$
l_{i j}=\left\{\begin{array}{ll}
\sum_{j=1, j \neq i}^{N} a_{i j} & i=j \\
-a_{i j} & i \neq j
\end{array} .\right.
$$

The in-degree and out-degree of node $v_{i}$ are defined as $d_{\text {in }}\left(v_{i}\right)=\sum_{j=1}^{N} a_{i j}$ and $d_{\text {out }}\left(v_{i}\right)=\sum_{j=1}^{N} a_{j i}$, respectively. A graph is balanced if and only if $d_{\text {in }}\left(v_{i}\right)=d_{\text {out }}\left(v_{i}\right), \forall v_{i} \in \mathcal{V}$. It is clear that an undirected graph is balanced. $(1 / \sqrt{N}) \mathbf{1}_{N}$ and $w_{1}^{T}$ $\left((1 / \sqrt{N}) \mathbf{1}_{N} w_{1}^{T}=1\right)$ are the right and left eigenvectors associated with the zero eigenvalue of $\mathcal{L}$, respectively. $w_{1}^{T}$ is $(1 / \sqrt{N}) \mathbf{1}_{N}^{T}$ if the digraph $\mathcal{D}$ is balanced. A class of piecewise right-continuous switching function $\sigma(t)($ in $\operatorname{short} \sigma):[0, \infty] \rightarrow \mathcal{P}=$ $\left\{1,2, \ldots, n_{v}\right\}$ is used to describe the switching topologies where $n_{v}$ is the total number of all possible communication graphs. We use $G^{\sigma(t)}$ to denote the communication graph at time $t$. An undirected graph is said to be connected if every two distinct nodes can be connected by a path where a path is a sequence of adjacent edges of the form $\left(v_{i_{1}}, v_{i_{2}}\right),\left(v_{i_{2}}, v_{i_{3}}\right), \ldots,\left(v_{i_{j-1}}, v_{i_{j}}\right)$ in which $i_{j} \in \mathcal{V}$. A directed graph is said to contain a directed spanning tree if it has at least one node from which there exist directed paths to all other nodes. The union of a collection of graphs $G_{1}, \ldots, G_{m}$ with the node set $\mathcal{V}$ is a graph denoted by $G_{1-m}$ with the same node set whose edge set is the union of the edge sets of all graphs. A collection of switching undirected or directed graphs is called jointly connected or uniformly jointly quasi-strongly connected [5] if, respectively, the union of the graphs is connected or has a directed spanning tree. We assume that there exists an infinite sequence of nonempty, bounded and contiguous time intervals $\left[t_{r}, t_{r+1}\right], r=0,1, \ldots$ with $t_{0}=0$ and $t_{r+1}-t_{r} \leq T_{1}$ for some constant $T_{1}>0$ such that the collection of switching graphs across each time interval is jointly connected or uniformly jointly quasi-strongly connected. It is also assumed that there is a sequence of non-overlapping subintervals $\left[t_{r_{0}}, t_{r_{1}}\right), \ldots,\left[t_{r_{j}}, t_{r_{j+1}}\right), \ldots,\left[t_{r_{m_{r}-1}}, t_{r_{m_{r}}}\right.$ ) 
where $t_{r}=t_{r_{0}}, t_{r+1}=t_{r_{r}}$ and $t_{r_{j+1}}-t_{r_{j}} \geq T_{2}, 0 \leq j<m_{r}-1$ for some integer $m_{r}$ and given constant $T_{2}$. The communication topology is supposed to be fixed on each time subinterval and switches at $t_{r_{j}}$.

\section{Problem statement}

Consider a team of $N$ high-order nonlinear agents which the dynamics of the ith one is as follows:

$$
x_{i}^{(n)}=f_{i}\left(\mathscr{X}_{i}\right)+b_{i} u_{i}+d_{i}(t)
$$

where $\mathscr{X}_{i}=\left[x_{i}, \dot{x}_{i}, \ldots, x_{i}^{(n-1)}\right]^{T} \in \mathbb{R}^{n}, u_{i} \in \mathbb{R}$ are system states and the control input, respectively. $x_{i}^{(h)} \in \mathbb{R}$ is the $h$ th-order state of the $i$ th agent and $x_{i}$ denotes the position. $f_{i}\left(\mathscr{X}_{i}\right) \in \mathbb{R}$ is an unknown nonlinear function. $b_{i}$ is an unknown nonzero constant gain with an unknown sign. $d_{i}(t)$ is an external bounded disturbance. In the sequel, we make the following assumptions:

Assumption 1. $u_{i}$ is gained by passing the designed input $v_{i}$ through a non-symmetric saturation constraint defined as

$$
u_{i}=\left\{\begin{array}{cll}
u_{\max _{i}} & \text { if } & v_{i}>u_{\max _{i}} \\
v_{i} & \text { if } & u_{\min _{i}} \leq v_{i} \leq u_{\max _{i}} \\
u_{\min _{i}} & \text { if } & v_{i}<u_{\min _{i}}
\end{array}\right.
$$

where $u_{\max _{i}}$ and $u_{\min _{i}}$ are known bounds of saturation nonlinearity.

Assumption 2. $\left|d_{i}\right| \leq D_{i}$ where $D_{i}$ is an unknown constant.

In order to tackle the unknown control direction, the Nussbaum-type function technique is exploited in this paper. $\mathcal{N}_{m}(\xi)$ is a Nussbaum function satisfies the two-sided properties [22]

$$
\begin{array}{ll}
\lim _{\theta \rightarrow \pm \infty} \sup & \frac{1}{\theta} \int_{0}^{\theta} \mathcal{N}_{m}(\xi) \mathrm{d} \xi=\infty \\
\lim _{\theta \rightarrow \pm \infty} \inf & \frac{1}{\theta} \int_{0}^{\theta} \mathcal{N}_{m}(\xi) \mathrm{d} \xi=-\infty .
\end{array}
$$

In our framework, we utilize $\mathcal{N}_{m}(\xi)=\xi^{2} \cos ((\pi / 2) \xi)$ as an example of an even Nussbaum-type function.

In this paper, we aim at designing a protocol for a group of agents with dynamics given in (2) such that all agents reach consensus on the position while the higher-order states converge to zero.

Lemma 1. Let us consider the first-order integral MAS. The dynamics of each agent is

$$
\dot{z}_{i}=-\sum_{j \in \mathcal{N}_{i}\left(G^{\sigma(t)}\right)} a_{i j}(t)\left(z_{i}-z_{j}\right)+w_{i}
$$

where $z_{i} \in \mathbb{R}$ is the state of the ith agent and $w_{i} \in \mathbb{R}$ is continuous function on $[0, \infty)$ except for at most a set with measure zero. For $\forall z_{i}(0)$ and $\forall w_{i}$ satisfying $w_{i} \in L_{1}$, then $z_{i} \in L_{\infty}$ and $\lim _{t \rightarrow \infty}\left(z_{i}(t)-z_{j}(t)\right)=0$ if $G^{\sigma(t)}$ is jointly connected or uniformly jointly quasi-strongly connected for respectively the undirected or directed graphs [5,43].

\section{Consensus for high-order nonlinear MASs}

Consensus for high-order nonlinear MASs is discussed in this section and the main result is presented in the form of a theorem.

Let us define the variable $s_{i}$ for the $i$ th agent as:

$$
s_{i}=\frac{1}{\gamma_{n}}\left(x_{i}^{(n-1)}+\gamma_{2} x_{i}^{(n-2)}+\ldots+\gamma_{n-1} \dot{x}_{i}+\gamma_{n} x_{i}\right)
$$

where the polynomial $s^{n-1}+\gamma_{2} s^{(n-2)}+\ldots+\gamma_{n-1} s+\gamma_{n}$ has roots in the open-left half plane.

To deal with the effect of the saturation constraint, the auxiliary system is defined as:

$$
\dot{\tau}_{i}=-\beta \tau_{i}+\frac{k_{i}(t)}{\gamma_{n}} \operatorname{sgn}\left(e_{i}\right)\left|\Delta u_{i}\right|
$$

where $\beta \in \mathbb{R}^{+}, e_{i}=\tilde{s_{i}}-z_{i}$, with $\tilde{s_{i}}=s_{i}-\tau_{i}$, and $\Delta u_{i}=u_{i}-v_{i}$, i.e.,

$$
\begin{aligned}
& \Delta u_{i}=\left\{\begin{array}{ccc}
u_{\max _{i}}-v_{i} & \text { if } & v_{i}>u_{\max _{i}} \\
0 & \text { if } & u_{\min _{i}} \leq v_{i} \leq u_{\max _{i}}, \\
u_{\min _{i}}-v_{i} & \text { if } & v_{i}<u_{\min _{i}}
\end{array}\right. \\
& \dot{k}_{i}=\alpha_{i 1}\left|e_{i} \Delta u_{i}\right|
\end{aligned}
$$


where $\alpha_{i 1} \in \mathbb{R}^{+}$and an auxiliary state $z_{i}$ is defined as

$$
\dot{z}_{i}=-\sum_{j \in \mathcal{N}_{i}\left(G^{\sigma(t)}\right)} a_{i j}(t)\left(\tilde{s}_{i}-\tilde{s}_{j}\right)
$$

with the initial condition $z_{i}(0)=x_{i}(0)$. Based on universal approximation theorem [14,24], any continuous function can be estimated with arbitrarily small error. Having this property in mind and exploiting the fact that $f_{i}\left(\mathscr{X}_{i}\right)+\Theta_{i}$ is continuous, we can say

$$
f_{i}\left(\mathscr{X}_{i}\right)+\Theta_{i}=W_{i}^{T} \psi_{i}\left(\Xi_{i}\right)+\epsilon_{i}\left(\Xi_{i}\right)
$$

where $\Theta_{i}=\sum_{i=1}^{n-1} \gamma_{i+1} x_{i}^{(n-i)}+\beta \gamma_{n} \tau_{i}, \Xi_{i}=\left[\mathscr{X}_{i}^{T}, \Theta_{i}\right]^{T}, \epsilon_{i}$ is a bounded approximation error, i.e., $\left|\epsilon_{i}\right| \leq \varepsilon_{i}$ where $\varepsilon_{i}$ is an unknown constant, $W_{i}$ is the bounded ideal weight vector with $l_{i}$ neurons, and $\psi_{i}=\left[\psi_{i 1}, \ldots, \psi_{i l_{i}}\right]^{T}$ is defined by

$$
\psi_{i j}\left(\Xi_{i}\right)=\exp \left(-\frac{\left(\Xi_{i}-\bar{\Xi}_{i j}\right)^{T}\left(\Xi_{i}-\bar{\Xi}_{i j}\right)}{v_{i j}^{2}}\right)
$$

for $j=1, \ldots, l_{i}$ in which $\bar{\Xi}_{i j}$ and $v_{i j}$ denote the center of the receptive field and the width of the Gaussian function, respectively. Since the ideal weights are unknown, we can approximate $f_{i}\left(\mathscr{X}_{i}\right)+\Theta_{i}$ by

$$
\hat{f}_{i}\left(\mathscr{X}_{i}\right)+\hat{\Theta}_{i}=\hat{W}_{i}^{T} \psi_{i}\left(\Xi_{i}\right) .
$$

Exploiting the above structure, our problem is converted to the consensus of a network of first-order systems (9) with a bounded perturbation and the stabilization problem of $e_{i}$. The idea behind such a structure is to first the error variable $e_{i}$ for each agent is driven to zero. To this end, we use the auxiliary system (6) to counteract the saturation effect, and neural network to approximate the unknown part of each agent dynamics. The Nussbaum gain parameter is also exploited to handle the unknown sign of the control direction. To deal with the time-varying disturbance and the approximation error of the developed neural network, we use adaptive sliding mode control. Since we have no knowledge about the bounds on the disturbance and approximation error, we make use of adaptive gains to estimate these bounds. After the variable $e_{i}$ goes to zero, we can show that the state of the auxiliary system $\tau_{i}$ is driven to zero for each agent while consensus on a common value for the variables $z_{i}$ is achieved, i.e., $z_{i} \rightarrow \sum_{i=1}^{N} z_{i}(0) / N$. This implies that $\tilde{s}_{i}=s_{i}$ and hence $s_{i} \rightarrow z_{i} \rightarrow$ $\sum_{i=1}^{N} z_{i}(0) / N$. Afterward, we prove that choosing the initial conditions as $z_{i}(0)=x_{i}(0)$ for the auxiliary system (9), one can have $x_{i} \rightarrow \sum_{i}^{N} x_{i}(0) / N$. Now we put our main result in the following theorem.

Theorem 1. Consider the MAS that is defined by (2). Provided that time-varying topology for graph $G^{\sigma(t)}$ is jointly connected or uniformly jointly quasi-strongly connected for the undirected or balanced directed graphs, respectively, the agents reach average consensus under the following protocol:

$$
v_{i}=\mathcal{N}_{m}\left(\xi_{i}\right)\left(\hat{W}_{i}(t)^{T} \psi_{i}\left(\Xi_{i}\right)+\varpi_{i}\right)
$$

where

$$
\begin{aligned}
& \dot{\xi}_{i}=\frac{1}{\gamma_{n}}\left(\hat{W}_{i}(t)^{T} \psi_{i}\left(\Xi_{i}\right)+\varpi_{i}\right) e_{i}, \\
& \dot{\hat{W}}_{i}=P_{i} \psi_{i}\left(\Xi_{i}\right) e_{i}, \\
& \varpi_{i}=\kappa_{i}(t) \operatorname{sgn}\left(e_{i}\right)+\gamma_{n} \sum_{j \in \mathcal{N}_{i}\left(G^{\sigma(t)}\right)} a_{i j}(t)\left(\tilde{s}_{i}-\tilde{s}_{j}\right),
\end{aligned}
$$

in which the adaptive gain matrix $P_{i}$ is symmetric positive definite and

$$
\dot{\kappa}_{i}=\alpha_{i 2}\left|e_{i}\right| .
$$

where $\alpha_{i 2} \in \mathbb{R}^{+}$.

Proof. The first time derivative of (5) is obtained by

$$
\dot{s}_{i}=\frac{1}{\gamma_{n}}\left(x_{i}^{(n)}+\gamma_{2} x_{i}^{(n-1)}+\ldots+\gamma_{n-1} \ddot{x}_{i}+\gamma_{n} \dot{x}_{i}\right)
$$

which by using (2) and $u_{i}=v_{i}+\Delta u_{i}$, it can be written by

$$
\dot{s}_{i}=\frac{1}{\gamma_{n}}\left(f_{i}\left(\mathscr{X}_{i}\right)+\sum_{i=1}^{n-1} \gamma_{i+1} x_{i}^{(n-i)}+b_{i} \Delta u_{i}+d_{i}\right)+\frac{b_{i}}{\gamma_{n}} v_{i} .
$$


By substituting $v_{i}$ from (12) into (17), one can get

$$
\dot{s}_{i}=\frac{1}{\gamma_{n}}\left(f_{i}\left(\mathscr{X}_{i}\right)+\sum_{i=1}^{n-1} \gamma_{i+1} x_{i}^{(n-i)}+b_{i} \Delta u_{i}+d_{i}\right)+\frac{b_{i} \mathcal{N}_{m}\left(\xi_{i}\right)}{\gamma_{n}}\left(\hat{W}_{i}(t)^{T} \psi_{i}\left(\mathscr{X}_{i}\right)+\varpi_{i}\right) .
$$

By taking derivative of $e_{i}=s_{i}-\tau_{i}-z_{i}$ and using (6), (9), and (18), one can obtain

$$
\begin{aligned}
\dot{e}_{i}= & \frac{1}{\gamma_{n}}\left(f_{i}\left(\mathscr{X}_{i}\right)+\sum_{i=1}^{n-1} \gamma_{i+1} x_{i}^{(n-i)}+\beta \gamma_{n} \tau_{i}+b_{i} \Delta u_{i}+d_{i}\right)+\frac{b_{i} \mathcal{N}_{m}\left(\xi_{i}\right)}{\gamma_{n}}\left(\hat{W}_{i}(t)^{T} \psi_{i}\left(\mathscr{X}_{i}\right)+\varpi_{i}\right) \\
& -\frac{k_{i}}{\gamma_{n}} \operatorname{sgn}\left(e_{i}\right)\left|\Delta u_{i}\right|+\sum_{j \in \mathcal{N}_{i}\left(G^{\sigma(t)}\right)} a_{i j}(t)\left(\tilde{s}_{i}-\tilde{s}_{j}\right) .
\end{aligned}
$$

By substituting (10) into (19), one can get

$$
\begin{aligned}
\dot{e}_{i}= & \frac{1}{\gamma_{n}}\left(W_{i}^{T} \psi_{i}\left(\Xi_{i}\right)+b_{i} \Delta u_{i}+d_{i}+\epsilon_{i}\right) \\
& +\frac{b_{i} \mathcal{N}_{m}\left(\xi_{i}\right)}{\gamma_{n}}\left(\hat{W}_{i}(t)^{T} \psi_{i}\left(\mathscr{X}_{i}\right)+\varpi_{i}\right) \\
& -\frac{k_{i}}{\gamma_{n}} \operatorname{sgn}\left(e_{i}\right)\left|\Delta u_{i}\right|+\sum_{j \in \mathcal{N}_{i}\left(G^{\sigma(t)}\right)} a_{i j}(t)\left(\tilde{s}_{i}-\tilde{s}_{j}\right) .
\end{aligned}
$$

Now, let us consider the positive definite function as $V_{i}=V_{i 1}+V_{i 2}$ with

$$
\begin{aligned}
& V_{i 1}=\frac{1}{2} e_{i}^{2}, \\
& V_{i 2}=\frac{1}{2 \gamma_{n}}\left(\tilde{W}_{i}^{T} P_{i}^{-1} \tilde{W}_{i}+\frac{1}{\alpha_{i 1}} \tilde{k}_{i}^{2}+\frac{1}{\alpha_{i 2}} \tilde{\kappa}_{i}^{2}\right),
\end{aligned}
$$

where $\tilde{W}_{i}(t)=\hat{W}_{i}(t)-W_{i}, \tilde{k}_{i}(t)=k_{i}(t)-\left|b_{i}\right|$, and $\tilde{\kappa}_{i}(t)=\kappa_{i}(t)-\left(D_{i}+\varepsilon_{i}+\beta \gamma_{n}\right)$. The reason for considering $V_{i 2}$ as a part of the overall Lyapunov function is to derive the adaptive laws for neural network weights and estimators $k_{i}$ and $\kappa_{i}$. Since the bounds on the signals $\left|b_{i}\right|$ and $D_{i}+\varepsilon_{i}+\beta \gamma_{n}$ are not known, the adaptive gains $k_{i}$ and $\kappa_{i}$ are respectively implemented to estimate these bounds.

By using (13) and taking the time derivative of $V_{i 1}$ along (20), one can conclude that

$$
\begin{aligned}
\dot{V}_{i 1} & =\frac{1}{\gamma_{n}}\left(W_{i}^{T} \psi_{i}\left(\Xi_{i}\right)+\gamma_{n} \sum_{j \in \mathcal{N}_{i}\left(G^{\sigma(t)}\right)} a_{i j}(t)\left(\tilde{s}_{i}-\tilde{s}_{j}\right)\right) e_{i} \\
& +b_{i} \mathcal{N}_{m}\left(\xi_{i}\right) \dot{\xi}_{i}-\frac{k_{i}}{\gamma_{n}}\left|e_{i} \Delta u_{i}\right|+\frac{b_{i}}{\gamma_{n}} e_{i} \Delta u_{i}+\frac{d_{i}+\epsilon_{i}}{\gamma_{n}} e_{i} .
\end{aligned}
$$

By subtracting and adding $\dot{\xi}_{i}$ to the right-hand side of (22) and using (13) and (15), it can be concluded that

$$
\begin{aligned}
\dot{V}_{i 1} & =\frac{1}{\gamma_{n}}\left(W_{i}^{T} \psi_{i}\left(\Xi_{i}\right)+\gamma_{n} \sum_{j \in \mathcal{N}_{i}\left(G^{\sigma(t)}\right)} a_{i j}(t)\left(\tilde{s}_{i}-\tilde{s}_{j}\right)\right) e_{i} \\
& +\dot{\xi}_{i}-\frac{1}{\gamma_{n}}\left(\hat{W}_{i}(t)^{T} \psi_{i}\left(\Xi_{i}\right)+\kappa_{i}(t) \operatorname{sgn}\left(e_{i}\right)\right. \\
& \left.+\gamma_{n} \sum_{j \in \mathcal{N}_{i}\left(G^{\sigma(t)}\right)} a_{i j}(t)\left(\tilde{s}_{i}-\tilde{s}_{j}\right)\right) e_{i}+b_{i} \mathcal{N}_{m}\left(\xi_{i}\right) \dot{\xi}_{i} \\
& -\frac{k_{i}}{\gamma_{n}}\left|e_{i} \Delta u_{i}\right|+\frac{b_{i}}{\gamma_{n}} e_{i} \Delta u_{i}+\frac{d_{i}+\epsilon_{i}}{\gamma_{n}} e_{i} \\
& =-\frac{1}{\gamma_{n}} \tilde{W}_{i}^{T} \psi_{i}\left(\Xi_{i}\right) e_{i}+\left(b_{i} \mathcal{N}_{m}\left(\xi_{i}\right)+1\right) \dot{\xi}_{i} \\
& -\frac{k_{i}}{\gamma_{n}}\left|e_{i} \Delta u_{i}\right|+\frac{b_{i}}{\gamma_{n}} e_{i} \Delta u_{i}-\frac{\kappa_{i}}{\gamma_{n}}\left|e_{i}\right|+\frac{d_{i}+\epsilon_{i}}{\gamma_{n}} e_{i} .
\end{aligned}
$$

By exploiting $e_{i} \Delta u_{i} \leq\left|e_{i} \Delta u_{i}\right|, e_{i} \leq\left|e_{i}\right|,\left|d_{i}\right| \leq D_{i},\left|\epsilon_{i}\right| \leq \varepsilon_{i}, \tilde{k}_{i}=k_{i}-\left|b_{i}\right|$, and $\kappa_{i}=\kappa_{i}-\left(D_{i}+\varepsilon_{i}+\beta \gamma_{n}\right)$, we have

$$
\begin{aligned}
\dot{V}_{i 1} \leq & -\frac{1}{\gamma_{n}} \tilde{W}_{i}^{T} \psi_{i}\left(\Xi_{i}\right) e_{i}+\left(b_{i} \mathcal{N}_{m}\left(\xi_{i}\right)+1\right) \dot{\xi}_{i} \\
& -\frac{\tilde{k}_{i}}{\gamma_{n}}\left|e_{i} \Delta u_{i}\right|-\frac{\tilde{\kappa}_{i}}{\gamma_{n}}\left|e_{i}\right|-\beta\left|e_{i}\right| .
\end{aligned}
$$

Please cite this article as: M.H. Rezaei et al., Adaptive consensus for high-order unknown nonlinear multiagent systems with unknown control directions and switching topologies, Information Sciences (2018), https://doi.org/10.1016/j.ins.2018.04.089 
Now we can obtain the time derivative of $V_{i}$ as

$$
\begin{aligned}
\dot{V}_{i} \leq & \frac{1}{\gamma_{n}} \tilde{W}_{i}^{T} P_{i}^{-1}\left(\dot{\tilde{W}}_{i}-P_{i} \psi_{i}\left(\Xi_{i}\right) e_{i}\right) \\
& +\left(b_{i} \mathcal{N}_{m}\left(\xi_{i}\right)+1\right) \dot{\xi}_{i}+\frac{\tilde{k}_{i}}{\gamma_{n} \alpha_{i 1}}\left(\dot{\tilde{k}}_{i}-\alpha_{i 1}\left|e_{i} \Delta u_{i}\right|\right)+\frac{\tilde{\kappa}_{i}}{\gamma_{n} \alpha_{i 2}}\left(\dot{\tilde{\kappa}}_{i}-\alpha_{i 2}\left|e_{i}\right|\right)-\beta\left|e_{i}\right| .
\end{aligned}
$$

Because $W_{i}, \theta_{i},\left|b_{i}\right|$, and $D_{i}+\varepsilon_{i}+\beta \gamma_{n}$ are constant, we have $\dot{\tilde{W}}_{i}=\dot{\hat{W}}_{i}, \dot{\tilde{\theta}}_{i}=\dot{\hat{\theta}}_{i}, \dot{\tilde{k}}=\dot{k}_{i}, \dot{\tilde{\kappa}}=\dot{\kappa}_{i}$, and hence, substituting (8), (14), and (16) into (23) yields

$$
\dot{V}_{i} \leq-\beta\left|e_{i}\right|+\left(b_{i} \mathcal{N}_{m}\left(\xi_{i}\right)+1\right) \dot{\xi}_{i}
$$

Hence, from (24), it follows that:

$$
\dot{V}_{i} \leq\left(b_{i} \mathcal{N}_{m}\left(\xi_{i}\right)+1\right) \dot{\xi}_{i} .
$$

By taking the time integral of both sides of (25), one can get

$$
0 \leq V_{i}(t) \leq V_{i}(0)+\int_{0}^{t} b_{i} \mathcal{N}_{m}\left(\xi_{i}(\tau)\right) \dot{\xi}_{i}(\tau) \mathrm{d} \tau+\xi_{i}(t)
$$

where $\xi_{i}(0)=0$. Since

$$
\int_{0}^{t} \mathcal{N}_{m}\left(\xi_{i}(\tau)\right) \dot{\xi}_{i}(\tau) \mathrm{d} \tau=\int_{0}^{\xi_{i}(t)} \mathcal{N}_{m}\left(\xi_{i}\right) \mathrm{d} \xi_{i},
$$

we have

$$
0 \leq V_{i}(t) \leq V_{i}(0)+\int_{0}^{\xi_{i}(t)} b_{i} \mathcal{N}_{m}\left(\xi_{i}\right) \mathrm{d} \xi_{i}+\xi_{i}(t)
$$

or equivalently

$$
-\xi_{i}(t)-V_{i}(0) \leq \int_{0}^{\xi_{i}(t)} b_{i} \mathcal{N}_{m}\left(\xi_{i}\right) \mathrm{d} \xi_{i} .
$$

According to (4), $\mathcal{N}_{m}\left(\xi_{i}\right)$ has the following properties:

$$
\lim _{\xi_{i}(t) \rightarrow \pm \infty} \sup \frac{1}{\xi_{i}(t)} \int_{0}^{\xi_{i}(t)} b_{i} \mathcal{N}_{m}\left(\xi_{i}\right) \mathrm{d} \xi_{i}=\infty
$$

and

$$
\lim _{\xi_{i}(t) \rightarrow \pm \infty} \inf \frac{1}{\xi_{i}(t)} \int_{0}^{\xi_{i}(t)} b_{i} \mathcal{N}_{m}\left(\xi_{i}\right) \mathrm{d} \xi_{i}=-\infty .
$$

By the method of contradiction, it can be proved that $\xi_{i}(t) \in L_{\infty}$. Suppose that $\xi_{i}(t)$ becomes unbounded, then there are two cases.

1. If $\xi_{i}(t) \rightarrow \infty$, then by utilizing (27), one can get

$$
\lim _{\xi_{i}(t) \rightarrow \infty}-\frac{\xi_{i}(t)+V_{i}(0)}{\xi_{i}(t)} \leq \frac{1}{\xi_{i}(t)} \int_{0}^{\xi_{i}(t)} b_{i} \mathcal{N}_{m}\left(\xi_{i}\right) \mathrm{d} \xi_{i}
$$

and it can be obtained that

$$
-1 \leq \lim _{\xi_{i}(t) \rightarrow \infty} \frac{1}{\xi_{i}(t)} \int_{0}^{\xi_{i}(t)} b_{i} \mathcal{N}_{m}\left(\xi_{i}\right) \mathrm{d} \xi_{i} .
$$

Easily observed that (30) contradicts with (29).

2. If $\xi_{i}(t) \rightarrow-\infty$, then by using (27), one can obtain

$$
\lim _{\xi_{i}(t) \rightarrow-\infty}-\frac{\xi_{i}(t)+V_{i}(0)}{\xi_{i}(t)} \geq \frac{1}{\xi_{i}(t)} \int_{0}^{\xi_{i}(t)} b_{i} \mathcal{N}_{m}\left(\xi_{i}\right) \mathrm{d} \xi_{i}
$$

and it can be concluded that

$$
-1 \geq \lim _{\xi_{i}(t) \rightarrow-\infty} \frac{1}{\xi_{i}(t)} \int_{0}^{\xi_{i}(t)} b_{i} \mathcal{N}_{m}\left(\xi_{i}\right) \mathrm{d} \xi_{i} .
$$

Again (31) contradicts with (28). 
Therefore, $\xi_{i}(t)$ is bounded, and $\int_{0}^{\xi_{i}(t)} b_{i} \mathcal{N}_{m}\left(\xi_{i}\right) \mathrm{d} \xi_{i}$ is also bounded. By exploiting (26), $V_{i}$ is bounded. According to $V_{i}=$ $V_{i 1}+V_{i 2}$, where $V_{i 1}$ and $V_{i 2}$ defined in (21), one can conclude that $e_{i}, \tilde{W}_{i}, \hat{W}_{i}, \tilde{k}_{i}, k_{i}, \tilde{\kappa}_{i}$, and $\kappa_{i}$ are bounded.

From (24), one can get

$$
\dot{V}_{i} \leq-\beta\left|e_{i}\right|+\left(b_{i} \mathcal{N}_{m}\left(\xi_{i}\right)+1\right) \dot{\xi}_{i}
$$

Then, the time integration of $(32)$ over $[0, \infty)$ becomes

$$
\int_{0}^{\infty}\left|e_{i}\right| \leq \frac{1}{\beta}\left(V_{i}(0)-V_{i}(\infty)+\int_{0}^{\xi_{i}(\infty)} b_{i} \mathcal{N}_{m}\left(\xi_{i}\right) \mathrm{d} \xi_{i}+\xi_{i}(\infty)\right)
$$

Since the right side of (33) is bounded, we can conclude that $e_{i} \in L_{1}$.

From (9) and $e_{i}=\tilde{s_{i}}-z_{i}$, one can get

$$
\dot{z}_{i}=-\sum_{j \in \mathcal{N}_{i}\left(G^{\sigma(t)}\right)} a_{i j}(t)\left(z_{i}-z_{j}\right)+w_{i}
$$

where

$$
w_{i}=-\sum_{j \in \mathcal{N}_{i}\left(G^{\sigma(t)}\right)} a_{i j}(t)\left(e_{i}-e_{j}\right) .
$$

Since $e_{i} \in L_{1}$ for $i=1, \ldots, N$, the equation (35) implies that $w_{i} \in L_{1}$. According to Lemma 1 , one can conclude that $z_{i}$ is bounded and $\lim _{t \rightarrow \infty}\left(z_{i}(t)-z_{j}(t)\right)=0$. Based on the facts that $z_{i} \in L_{\infty}, e_{i} \in L_{\infty}$, and $e_{i}=\tilde{s_{i}}-z_{i}$, the boundedness of $\tilde{s_{i}}$ can be concluded. According to $\tilde{s}_{i} \in L_{\infty}$ for $i=1, \ldots, N, \hat{W}_{i}, \kappa_{i}, \xi_{i} \in L_{\infty}, 0<\psi_{i j}\left(\Xi_{i}\right) \leq 1$, and (12), it can be said that $v_{i}$ is bounded. Since $v_{i} \in L_{\infty}$ and (7), $\Delta u_{i}$ is bounded. Based on $\tilde{s}_{i} \in L_{\infty}$ for $i=1, \ldots, N, \Delta u_{i}, \xi_{i}, \hat{W}_{i}, \kappa_{i}, e_{i}, k_{i} \in L_{\infty},\left|d_{i}\right| \leq D_{i},\left|\epsilon_{i}\right| \leq \varepsilon_{i}$, $0<\psi_{i j}\left(\Xi_{i}\right) \leq 1$, and (20), one can conclude that $\dot{e}_{i}$ is bounded. Barbalat's lemma can also be applied in this case because $e_{i}$ and $\dot{e}_{i}$ are bounded and $e_{i} \in L_{1}$. Therefore, one can conclude that

$$
\lim _{t \rightarrow \infty} e_{i}(t)=0 .
$$

To proof the boundedness of $\tau_{i}$, let us rewrite (6) as

$$
\dot{\tau}_{i}=-\beta \tau_{i}+h_{i}
$$

where $h_{i}=\left(k_{i} / \gamma_{n}\right) \operatorname{sgn}\left(e_{i}\right)\left|\Delta u_{i}\right|$. Since $k_{i}, \Delta u_{i} \in L_{\infty}, h_{i}$ is bounded. Hence, (36) can be regarded as a linear systems with bounded input $h_{i}$. It is obvious that $\tau_{i} \in L_{\infty}$ since input $h_{i}$ is bounded. Based on $\lim _{t \rightarrow \infty} e_{i}(t)=0$ and $h_{i}=$ $\left(k_{i} / \gamma_{n}\right) \operatorname{sgn}\left(e_{i}\right)\left|\Delta u_{i}\right|$, one can conclude that $\lim _{t \rightarrow \infty} h_{i}(t)=0$, which results in $\lim _{t \rightarrow \infty} \tau_{i}(t)=0$. Based on $\lim _{t \rightarrow \infty} \tau_{i}(t)=0$, $\lim _{t \rightarrow \infty} e_{i}(t)=0$, and $e_{i}=s_{i}-\tau_{i}-z_{i}$, one can get

$$
\lim _{t \rightarrow \infty} s_{i}(t)=\lim _{t \rightarrow \infty} z_{i}(t) \text {. }
$$

Now, (34) can be described for the whole MAS as:

$$
\dot{z}=-\mathcal{L}_{G^{\sigma(t)}} z-\mathcal{L}_{G^{\sigma(t)}} e
$$

where $z=\left[z_{1}, z_{2}, \ldots, z_{N}\right]^{T}$ and $e=\left[e_{1}, e_{2}, \ldots, e_{N}\right]^{T}$. Since $G^{\sigma(t)}$ is balanced at any time instant, one have $\mathbf{1}_{N}^{T} \mathcal{L}_{G^{\sigma}(t)}=\mathbf{0}_{N}^{T}$. Based on this and by multiplying both sides of (37) by $\mathbf{1}_{N}^{T}$, one can conclude that $\mathbf{1}_{N}^{T} \dot{z}=\mathbf{0}_{N}^{T}$. Then, taking integration yields $\mathbf{1}_{N}^{T} z(t)=$ $\mathbf{1}_{N}^{T} z(0)$. Since $\lim _{t \rightarrow \infty} s_{i}(t)=\lim _{t \rightarrow \infty} z_{i}(t), \lim _{t \rightarrow \infty} z_{1}(t)=z_{2}(t)=\ldots=z_{N}(t)$, and $z_{i}(0)=x_{i}(0)$, one can conclude that

$$
\lim _{t \rightarrow \infty} s_{i}(t)=\lim _{t \rightarrow \infty} z_{i}(t)=\frac{1}{N} \sum_{i=1}^{N} x_{i}(0) .
$$

By taking the Laplace transform of (5) and after some mathematical manipulations, one can obtain

$$
X_{i}(s)=\frac{1}{s^{n-1}+\gamma_{2} s^{n-2}+\ldots+\gamma_{n}} \times\left(\gamma_{n} S_{i}(s)+\sum_{k=1}^{n-1} s^{n-1-k} x_{i}^{(k-1)}(0)+\sum_{h=2}^{n-1} \sum_{k=1}^{n-h} \gamma_{h} s^{n-h-k} x_{i}^{(k-1)}(0)\right)
$$

where $X_{i}(s)$ and $S_{i}(s)$ denote Laplace transform of $x_{i}(t)$ and $s_{i}(t)$, respectively. By using $(38), \lim _{t \rightarrow \infty} s_{i}(t)=(1 / N) \sum_{i=1}^{N} x_{i}(0)$, the final value theorem, i.e., $\lim _{t \rightarrow \infty} x_{i}(t)=\lim _{s \rightarrow 0} s X_{i}(s)$ and $\lim _{s \rightarrow 0} s S_{i}(s)=\lim _{t \rightarrow \infty} s_{i}(t)$, and the fact that the polynomial $s^{n-1}+\gamma_{2} s^{n-2}+\ldots+\gamma_{n}$ has roots in the open-left half plane, one can conclude that

$$
\lim _{t \rightarrow \infty} x_{i}(t)=\frac{1}{N} \sum_{i=1}^{N} x_{i}(0)
$$

and therefore, $\lim _{t \rightarrow \infty} x_{i}^{(k)}(t)=0, k \in\{1, \ldots, n-1\}$, and the proof is completed.

Remark 1. Exploiting the auxiliary systems (6) along with introducing variables $s_{i}, e_{i}$, and $z_{i}$, in the proposed control scheme, the group consensus of the high-order nonlinear MAS with unknown nonlinearities, disturbances and unknown 
Table 2

Initial states of the agents .

\begin{tabular}{llll}
\hline Agent & $\phi_{i 1}, \phi_{i 2}, \phi_{i 3}, \phi_{i 4}$ & Agent & $\phi_{i 1}, \phi_{i 2}, \phi_{i 3}, \phi_{i 4}$ \\
\hline 1 & $1,0.5,1,0.8$ & 4 & $0,0.2,0.5,0.2$ \\
2 & $0.5,0.5,0.2,0.1$ & 5 & $0.2,5,0.2,0,-0.5,1$ \\
3 & $1,0,2,0.5$ & 6 & $-0.5,0.8,0.5,-0.4$ \\
\hline
\end{tabular}

control direction is decoupled to the two tasks: consensus of the first-order MASs (9) and the stabilization control of the unknown nonlinear system with input saturation and external disturbance for $e_{i}$ with the dynamics obtained in (19). As it is discussed in $[1,3,13,30]$, extending conventional Nussbaum gain technique to the cooperative task is quite involved. Having introduced the proposed structure, we facilitate extending the Nussbaum-type function for an individual system to the cooperative case and also relax all the limiting conditions. Our method just requires the control effects to have non-zero values.

Remark 2. In the proposed framework, each agent is supposed to transmit the signal $\tilde{s}_{i}$ to its neighbors. This signal can be calculated online on each agent.

Remark 3. It is noteworthy that an important issue, which is very important and crucial especially in MASs is eventtriggered control. In this approach, the sensors and controllers are updated when a specific event happens. This framework comes up with several advantages such as reducing the communication bandwidth and the control effort. Because, in practice, the control systems and embedded sensors are resource constrained, the event triggered control holds promising potential in practical implementation of distributed control systems and hence, worth to be taken into account in this context. The readers are referred to [8-10,15-17] for more information.

Remark 4. The final value of the agreement is dependent on the initial conditions of the auxiliary systems (9), i.e., $x_{i} \rightarrow \sum_{i=1}^{N} z_{i}(0) / N$. Therefore, the average consensus is accomplished by letting $z_{i}(0)=x_{i}(0)$. In the directed case, the graph should be uniformly jointly quasi-strongly connected and balanced in order to average consensus is achieved. If the network topology is only uniformly jointly quasi-strongly connected and not balanced, then the consensus is still acquired but the final value of the agreement is not the average of the initial conditions.

\section{Simulation results}

Cooperation of the manipulators plays a key role in the assembly automation and production processes with high flexibility. One of the typical task in force control is the grasping task via robot manipulators in which all manipulators are required to reach a common configuration. To study the effectiveness of the presented control, we conduct numerical simulations for consensus of multiple single-link flexible joint manipulators. This is done by applying both our method and that of [37]. Consider a group of six single-link flexible joint manipulators with DC motor actuators which the dynamics of the $i$ th one is governed by the following equations [37]

$$
\text { Motor } \begin{cases}\dot{\phi}_{1 i}= & \phi_{2 i} \\ \dot{\phi}_{2 i}= & g_{i}\left(\phi_{1 i}, \phi_{2 i}, \phi_{3 i}\right)+\rho_{i} u_{i}+d_{i}\end{cases}
$$

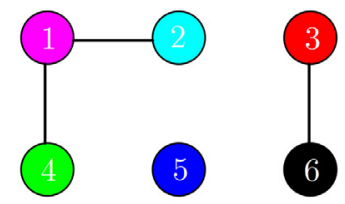

(a) Topology 1.

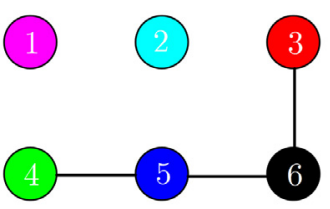

(c) Topology 3.

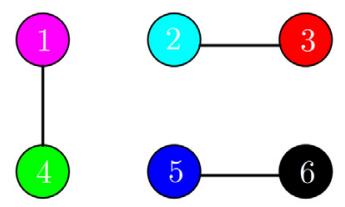

(b) Topology 2 .

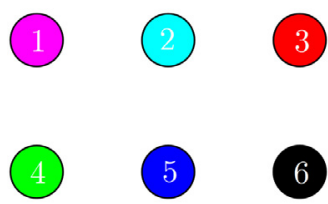

(d) Topology 4 .

Fig. 1. Four communication topologies of the six agents. 


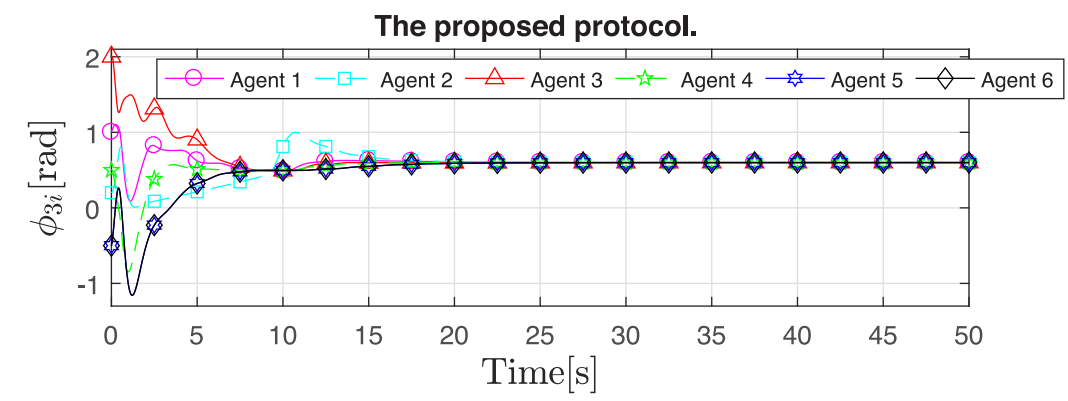

The presented protocol in [38].

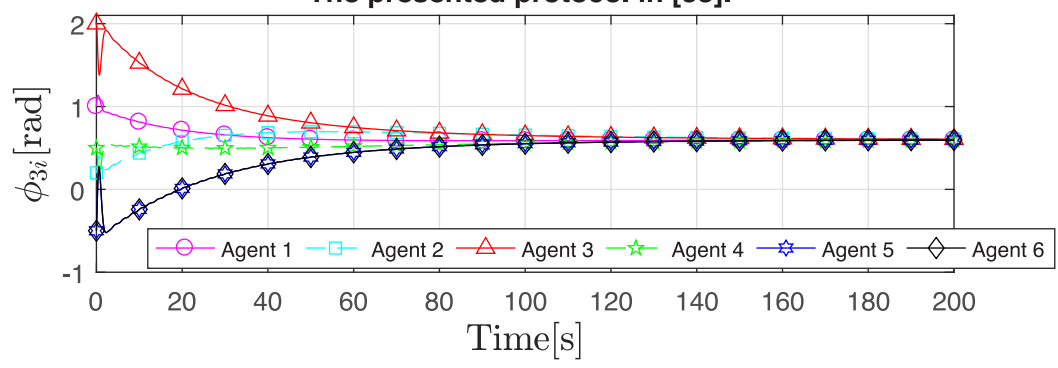

Fig. 2. Trajectories of the six joints angular rotations.

$$
\text { Joint } \begin{cases}\dot{\phi}_{3 i}= & \phi_{4 i} \\ \dot{\phi}_{4 i}= & 19.5\left(\phi_{1 i}-\phi_{3 i}\right)-3.33 \sin \left(\phi_{3 i}\right)\end{cases}
$$

where $\phi_{1 i}$ and $\phi_{2 i}$ denote, respectively, the angular rotation and angular velocity of the manipulator motor, $\phi_{3 i}$ and $\phi_{4 i}$ denote the angular rotation and angular velocity of the manipulator joint, respectively, $u_{i}$ is the control input, $g_{i}\left(\phi_{1 i}, \phi_{2 i}, \phi_{3 i}\right)=$ 48.6 $\left(\phi_{3 i}-\phi_{1 i}\right)-1.25 \phi_{2 i}$, and $\rho_{i}=21.6$. In the process of control design, in [37], $\rho_{i}$ is supposed to be an unknown positive constant while in our method it is assumed that $\rho_{i}$ is an unknown constant. As another difference, in [37], $g_{i}\left(\phi_{1 i}, \phi_{2 i}\right.$, $\phi_{3 i}$ ) is described by a known nonlinear regressor vector multiplied by an unknown constant parameter vector. However, in our framework, this nonlinear function is considered to be completely unknown. $d_{i}$ is chosen to be 0.1 isin $(t)$. Considering $x_{i}=\phi_{3 i}$, one can have

$$
\begin{aligned}
x_{i} & =\phi_{3 i} \\
\dot{x}_{i} & =\phi_{4 i} \\
\ddot{x}_{i} & =19.5 \phi_{1 i}-19.5 \phi_{3 i}-3.33 \sin \left(\phi_{3 i}\right) \\
\dddot{x}_{i} & =19.5 \phi_{2 i}-19.5 \phi_{4 i}-3.33 \phi_{4 i} \cos \left(\phi_{3 i}\right) .
\end{aligned}
$$

Now the equation of each manipulator can be transformed to the following fourth-order dynamics

$$
x_{i}^{(4)}=f_{i}\left(\mathscr{X}_{i}\right)+b_{i} u_{i}+19.5 d_{i}
$$

with $b_{i}=19.5 \rho_{i}, \mathscr{X}_{i}=\left[x_{i}, \dot{x}_{i}, \ddot{x}_{i}, \dddot{x}_{i}\right]^{T}$. In [37], $f_{i}\left(\mathscr{X}_{i}\right)$ is described by a known nonlinear regressor vector

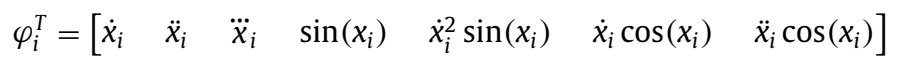

multiplied by an unknown constant parameter vector $\psi_{i} \in \mathbb{R}^{7}$ as $f_{i}\left(\mathscr{X}_{i}\right)=\varphi_{i}^{T} \psi_{i}$. However, in our framework, $f_{i}\left(\mathscr{X}_{i}\right)$ is completely unknown function as:

$$
f_{i}\left(\mathscr{X}_{i}\right)=19.5 g_{i}-19.5 \ddot{x}_{i}+3.33 \dot{x}_{i}^{2} \sin \left(x_{i}\right)-3.33 \ddot{x}_{i} \cos \left(x_{i}\right) .
$$

The interaction topology of the manipulators via which the agents exchange their information is jointly connected. The network topology switches between the four graphs shown in Fig. 1. $\sigma(t)=\operatorname{fix}(\bmod (2 t, 4)+1)$. The initial conditions, $\left(\phi_{i 1}(0), \phi_{i 2}(0), \phi_{i 3}(0), \phi_{i 4}(0)\right)$, are given in Table 2. The controller parameters of the proposed control scheme are selected as $\epsilon=0.02, s^{3}+\gamma_{2} s^{2}+\gamma_{3} s+\gamma_{4}=(s+3)^{3}, \beta=5, P_{i}=100 I, \alpha_{i 1}=\alpha_{i 2}=1, \kappa_{i}(0)=k_{i}(0)=10$, and $\hat{W}_{i j}(0) \in[-500,500], i=$ $1, \ldots, 6, j=1, \ldots, 25$. The input saturation limit is $[-10,12]$, i.e., $-10 \leq u_{i}(t) \leq 12$. The RBFNN contains 25 nodes with centers evenly spaced in $[-2,2] \times[-0.5,0.5] \times[-2,2] \times[-0.5,0.5] \times[-25,25]$, and widths 0.5 . One approach to reduce chattering is to use a continuous approximation of the $\operatorname{sgn}(x)$ function [25]. We approximate the $\operatorname{sgn}($.$) function by the sat(.)$ function described as

$$
\operatorname{sat}\left(\frac{x}{\epsilon}\right)=\left\{\begin{array}{cc}
\operatorname{sgn}(x) & |x| \geq \epsilon \\
\frac{x}{\epsilon} & |x|<\epsilon
\end{array}\right.
$$

Please cite this article as: M.H. Rezaei et al., Adaptive consensus for high-order unknown nonlinear multiagent systems with unknown control directions and switching topologies, Information Sciences (2018), https://doi.org/10.1016/j.ins.2018.04.089 

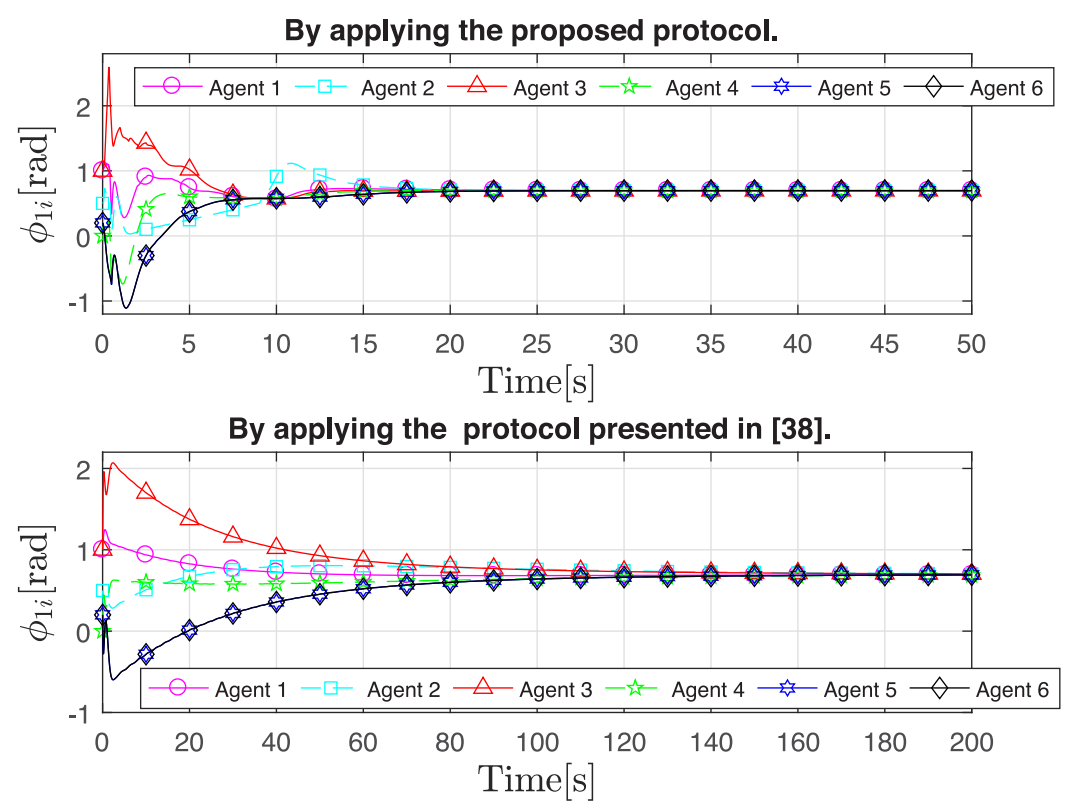

Fig. 3. Trajectories of the six motors angular rotations.
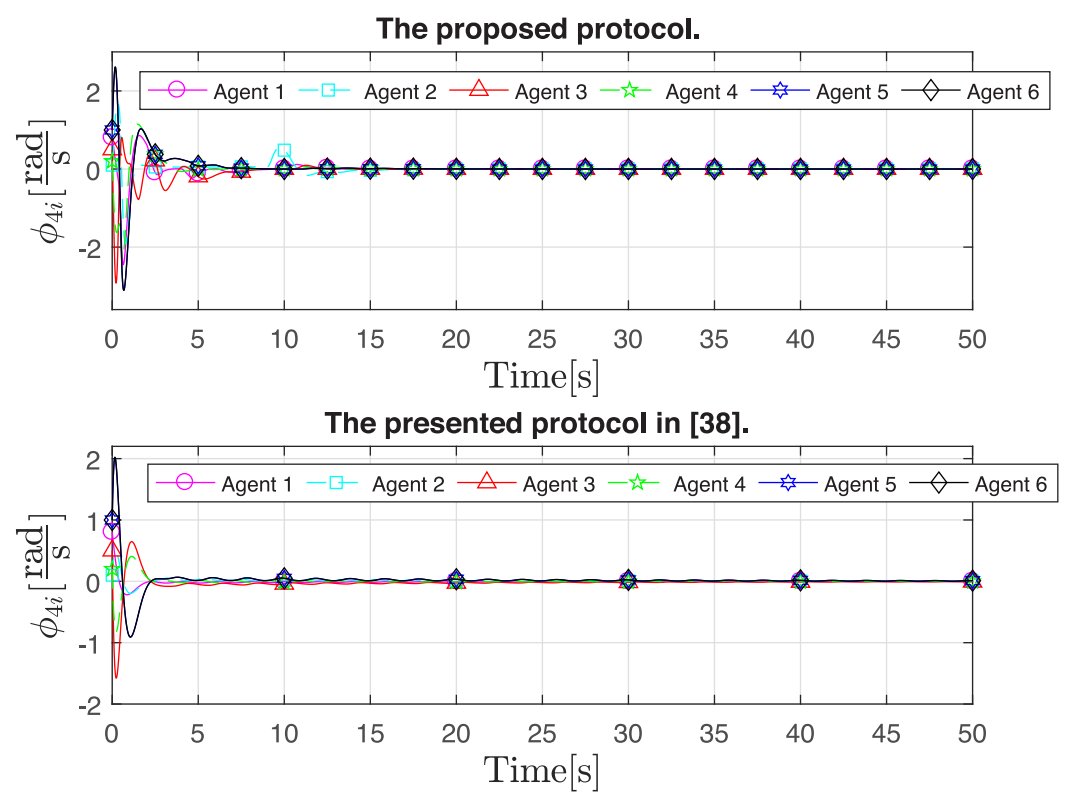

Fig. 4. Trajectories of the six joints angular velocities.

To conduct simulations for the approach of [37], the control parameters are selected the same as those in the numerical example of that paper.

The trajectories of the joints angular rotations are shown in Fig. 2, which clearly visualizes the process of group consensus. The trajectories of the motors angular rotations are shown in Fig. 3. As we can see from Figs. 2 and 3 the responses of our approach are much faster than that of [37]. The convergence of angular velocities of the joints and the motors to zero are presented in Figs. 4 and 5, respectively. As it is obvious from Fig. 6, in our framework compared to [37], the cooperative goal is achieved with much less control effort which highlights the practical importance of the presented control scheme. 


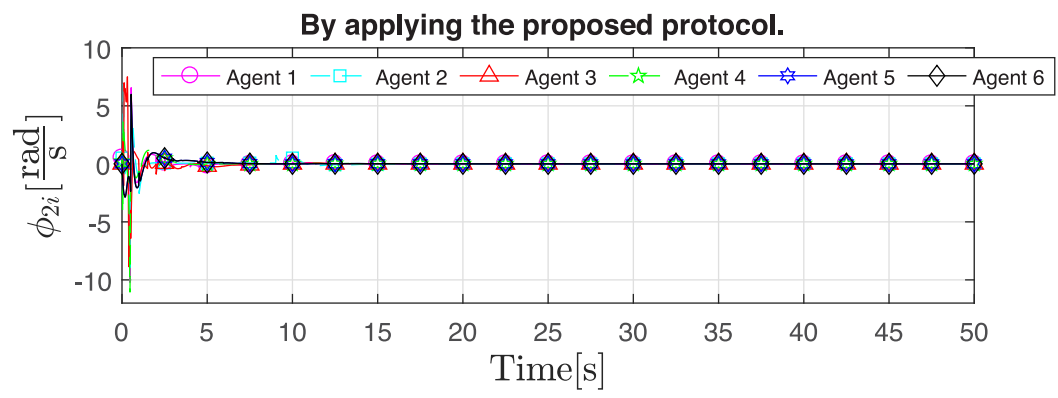

By applying the protocol presented in [38].

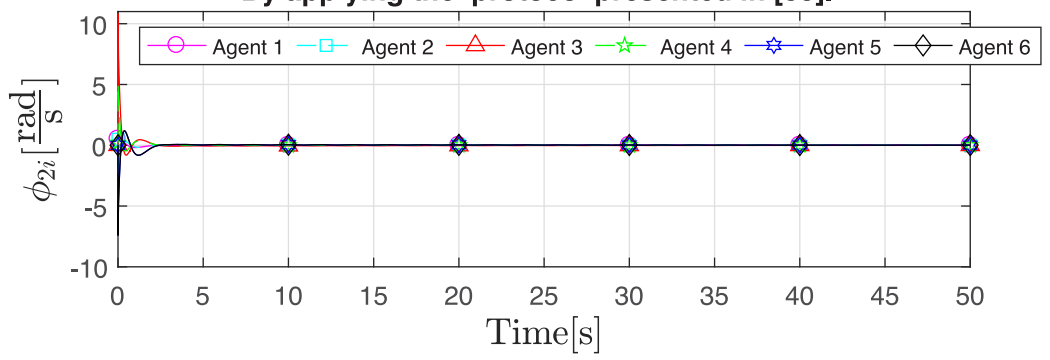

Fig. 5. Trajectories of the six motors angular velocities.

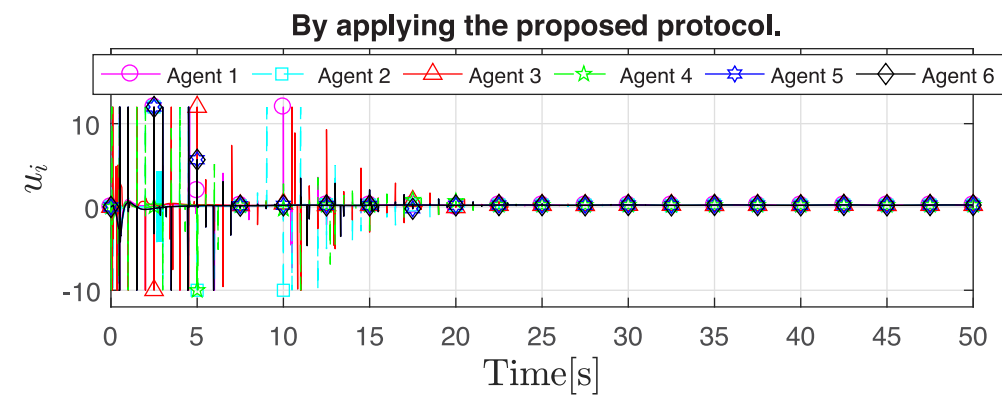

By applying the protocol presented in [38].

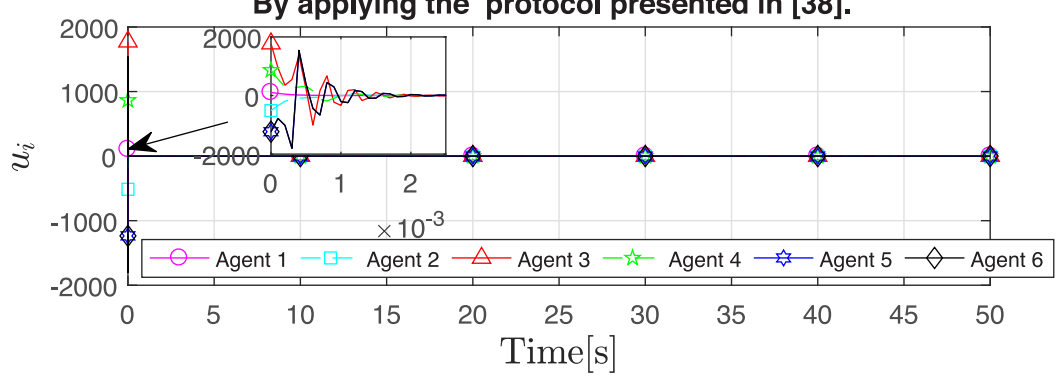

Fig. 6. Control input signals.

\section{Conclusion}

In this paper, we studied the consensus problem of unknown high-order nonlinear MASs with input saturation, unknown control direction, time-varying disturbance, and switching topologies. The problem was first decoupled to the consensus of a group of first-order MASs and the stabilization control. Furthermore, respectively, the Nussbaum-type function method, auxiliary systems, and radial basis function neural networks were used to deal with unknown control directions, input saturation, and the unknown nonlinearities existed in the agent dynamics. The approximation error of the developed neural network along with the disturbance was counteracted using an adaptive sliding mode control scheme. The asymptotic stability of the overall system was established using Lyapunov function theory. Comparative simulations were also performed for a group of single-link flexible joint manipulators. As our future research, we plan to extend the design to the event-triggered control problem.

Please cite this article as: M.H. Rezaei et al., Adaptive consensus for high-order unknown nonlinear multiagent systems with unknown control directions and switching topologies, Information Sciences (2018), https://doi.org/10.1016/j.ins.2018.04.089 


\section{References}

[1] C. Chen, C. Wen, Z. Liu, K. Xie, Y. Zhang, C.P. Chen, Adaptive consensus of nonlinear multiagent systems with non-identical partially unknown control directions and bounded modelling errors, IEEE Trans. Autom. Control 62 (9) (2017) 4654-4659.

[2] S. Chen, D.W. Ho, Consensus control for multiple auvs under imperfect information caused by communication faults, Inf. Sci. 370 (2016) $565-577$.

[3] W. Chen, X. Li, W. Ren, C. Wen, Adaptive consensus of multiagent systems with unknown identical control directions based on a novel nussbaum-type function, IEEE Trans. Autom. Control 59 (7) (2014) 1887-1892.

[4] Y. Chen, Y. Shi, Consensus for linear multiagent systems with time-varying delays: a frequency domain perspective, IEEE Trans. Cybern. 47 (8) (2017) 2143-2150.

[5] L. Cheng, H. Wang, Z.G. Hou, M. Tan, Reaching a consensus in networks of high-order integral agents under switching directed topologies, Int. J. Syst. Sci. 47 (8) (2016) 1966-1981.

[6] M.A. Dehghani, M.B. Menhaj, Communication free leader-follower formation control of unmanned aircraft systems, Robot. Auton. Syst. 80 (2016) 69-75.

[7] C. Deng, G.H. Yang, Consensus of linear multiagent systems with actuator saturation and external disturbances, IEEE Trans. Circuits Syst. II, Exp. Briefs. 64 (3) (2017) 284-288.

[8] D. Ding, Z. Wang, D.W. Ho, G. Wei, Observer-based event-triggering consensus control for multiagent systems with lossy sensors and cyber-attacks, IEEE Trans. Cybern. 47 (8) (2017) 1936-1947.

[9] D. Ding, Z. Wang, B. Shen, G. Wei, Event-triggered consensus control for discrete-time stochastic multi-agent systems: the input-to-state stability in probability, Automatica 62 (2015) 284-291.

[10] L. Ding, Q.L. Han, X. Ge, X.M. Zhang, An overview of recent advances in event-triggered consensus of multiagent systems, IEEE Trans. Cybern. 48 (4) (2018) 2168-2267.

[11] L. Ding, Q.L. Han, G. Guo, Network-based leader-following consensus for distributed multi-agent systems, Automatica 49 (7) (2013) $2281-2286$.

[12] L. Ding, Q.L. Han, L.Y. Wang, E. Sindi, Distributed cooperative optimal control of dc microgrids with communication delays, IEEE Trans. Ind. Informat (2018), doi:10.1109/TII.2018.2799239.

[13] Z. Ding, Adaptive consensus output regulation of a class of nonlinear systems with unknown high-frequency gain, Automatica 51 (2015) $348-355$.

[14] S.S. Ge, C.C. Hang, T.H. Lee, T. Zhang, Stable Adaptive Neural Network Control, 13, Springer Science \& Business Media, 2013.

[15] X. Ge, Q.L. Han, D. Ding, X.M. Zhang, B. Ning, A survey on recent advances in distributed sampled-data cooperative control of multi-agent systems, Neurocomputing 275 (2018) 1684-1701.

[16] X. Ge, Q.L. Han, F. Yang, Event-based set-membership leader-following consensus of networked multi-agent systems subject to limited communication resources and unknown-but-bounded noise, IEEE Trans. Ind. Electron. 64 (6) (2017) 5045-5054.

[17] X. Ge, F. Yang, Q.L. Han, Distributed networked control systems: a brief overview, Inf. Sci. 380 (2017) 117-131.

[18] Y. Guan, Q.L. Han, X. Ge, On asynchronous event-triggered control of decentralized networked systems, Inf. Sci. 425 (2018) $127-139$.

[19] W. He, G. Chen, Q.L. Han, F. Qian, Network-based leader-following consensus of nonlinear multi-agent systems via distributed impulsive control, Inf. Sci. 380 (2017) 145-158.

[20] Y. Hong, L. Gao, D. Cheng, J. Hu, Lyapunov-based approach to multiagent systems with switching jointly connected interconnection, IEEE Trans. Autom. Control 52 (5) (2007) 943-948.

[21] C. Hua, Y. Li, X. Guan, Leader-following consensus for high-order nonlinear stochastic multiagent systems, IEEE Trans. Cybern. 47 (8) (2017) 1882-1891.

[22] A. Ilchmann, Non-identifier-Based High-Gain Adaptive Control, Springer, London, 1993.

[23] M. Kabiri, H. Atrianfar, M.B. Menhaj, Formation control of vtol uav vehicles under switching directed interaction topologies with disturbance rejection, Int. J. Control 91 (1) (2018) 33-44.

[24] B. Karimi, M.B. Menhaj, Non-affine nonlinear adaptive control of decentralized large-scale systems using neural networks, Inf. Sci. 180 (17) (2010) 3335-3347.

[25] H.K. Khalil, J. Grizzle, Nonlinear Systems, Vol. 3, Prentice Hall, Upper Saddle River, 2002.

[26] F. Li, Y. Wu, W. Pedrycz, A modified consensus model in group decision making with an allocation of information granularity, IEEE Trans. Fuzzy Syst. (2018), doi:10.1109/TFUZZ.2018.2793885.

[27] H. Li, X. Liao, T. Huang, Y. Wang, Q. Han, T. Dong, Algebraic criteria for second-order global consensus in multi-agent networks with intrinsic nonlinear dynamics and directed topologies, Inf. Sci. 259 (2014) 25-35.

[28] H. Liu, H.R. Karimi, S. Du, W. Xia, C. Zhong, Leader-following consensus of discrete-time multiagent systems with time-varying delay based on large delay theory, Inf. Sci. 417 (2017) 236-246.

[29] K. Liu, Z. Ji, W. Ren, Necessary and sufficient conditions for consensus of second-order multiagent systems under directed topologies without global gain dependency, IEEE Trans. Cybern. 47 (8) (2017) 2089-2098.

[30] L. Liu, Adaptive cooperative output regulation for a class of nonlinear multiagent systems, IEEE Trans. Autom. Control 60 (6) (2015) $1677-1682$.

[31] X. Ma, F. Sun, H. Li, B. He, Neural-network-based sliding-mode control for multiple rigid-body attitude tracking with inertial information completely unknown, Inf. Sci. 400 (2017) 91-104.

[32] R.D. Nussbaum, Some remarks on a conjecture in parameter adaptive control, Syst. Control Lett. 3 (5) (1983) $243-246$.

[33] R. Olfati-Saber, Flocking for multiagent dynamic systems: algorithms and theory, IEEE Trans. Autom. Control 51 (3) (2006) $401-420$.

[34] R. Olfati-Saber, R.M. Murray, Consensus problems in networks of agents with switching topology and time-delays, IEEE Trans. Autom. Control 49 (9) (2004) 1520-1533.

[35] J. Qin, W. Fu, H. Gao, W.X. Zheng, Distributed k-means algorithm and fuzzy c-means algorithm for sensor networks based on multiagent consensus theory, IEEE Trans. Cybern. 47 (3) (2017) 772-783.

[36] J. Qin, W. Fu, W.X. Zheng, H. Gao, On the bipartite consensus for generic linear multiagent systems with input saturation, IEEE Trans. Cybern. 47 (8) (2017) 1948-1958.

[37] H. Rezaee, F. Abdollahi, Adaptive stationary consensus protocol for a class of high-order nonlinear multiagent systems with jointly connected topologies, Int. J. Robust Nonlin. Control 27 (9) (2017) 1677-1689.

[38] H. Rezaee, F. Abdollahi, Consensus problem over high-order multiagent systems with uncertain nonlinearities under deterministic and stochastic topologies, IEEE Trans. Cybern. 47 (8) (2017) 2079-2088.

[39] M.H. Rezaei, M.B. Menhaj, Stationary average consensus for high-order multi-agent systems, IET Control Theory Appl. 11 (5) (2017) 723-731.

[40] M.H. Rezaei, M.B. Menhaj, Stationary average consensus protocol for a class of heterogeneous high-order multi-agent systems with application for aircraft, Int. J. Syst. Sci. 49 (2) (2018) 284-298.

[41] M.H. Rezaei, M.B. Menhaj, Adaptive output stationary average consensus for heterogeneous unknown linear multi-agent systems, IET Control Theory Appl. (2018), doi:10.1049/iet-cta.2017.0877.

[42] G. Shi, K.H. Johansson, Robust consensus for continuous-time multiagent dynamics, SIAM J. Control Optim. 51 (5) (2013) $3673-3691$.

[43] H. Su, Y. Qiu, L. Wang, Semi-global output consensus of discrete-time multi-agent systems with input saturation and external disturbances, ISA Trans. 67 (2017) 131-139.

[44] B. Wang, J. Wang, B. Zhang, X. Li, Global cooperative control framework for multiagent systems subject to actuator saturation with industrial applications, IEEE Trans. Syst. Man Cybern. Syst. 47 (7) (2017) 1270-1283.

[45] W. Wang, S. Tong, Adaptive fuzzy bounded control for consensus of multiple strict-feedback nonlinear systems, IEEE Trans. Cybern. 48 (2) (2018) $522-531$. 
M.H. Rezaei et al./Information Sciences 000 (2018) 1-14

[46] Y. Yang, D. Yue, C. Dou, Distributed adaptive output consensus control of a class of heterogeneous multiagent systems under switching directed topologies, Inf. Sci. 345 (2016) 294-312.

[47] G. Zhang, J. Qin, W.X. Zheng, Y. Kang, Fault-tolerant coordination control for second-order multi-agent systems with partial actuator effectiveness, Inf. Sci. 423 (2018) 115-127.

[48] A.M. Zou, A.H. de Ruiter, K.D. Kumar, Distributed attitude synchronization control for a group of flexible spacecraft using only attitude measurements, Inf. Sci. 343 (2016) 66-78.

Please cite this article as: M.H. Rezaei et al., Adaptive consensus for high-order unknown nonlinear multiagent systems with unknown control directions and switching topologies, Information Sciences (2018), https://doi.org/10.1016/j.ins.2018.04.089 